\title{
Consumo de álcool e violência física entre adolescentes: quem é o preditor?
}

\author{
Alcohol consumption and physical violence among adolescents: \\ which is the predictor?
}

Amanda Pacheco de Carvalho ${ }^{1}$

Thaís Carine da Silva ${ }^{1}$

Paula Andrea de Melo Valença ${ }^{1}$

Carolina da Franca Bandeira Ferreira Santos ${ }^{1}$

Viviane Colares ${ }^{1}$

Valdenice Aparecida de Menezes ${ }^{1}$

\footnotetext{
${ }^{1}$ Programa de Hebiatria, Faculdade de Odontologia, Universidade de

Pernambuco. Av. General Newton Cavalcanti 1650, Camaragibe. 51021-350 Camaragibe PE Brasil. amandapdc@gmail.com
}

\begin{abstract}
The scope of this integrative review was to investigate the association between alcohol consumption and physical violence in the literature, with an emphasis on identifying the predictor between them. A search was conducted in the Lilacs, Medline and SciELO databases, adopting "violence," "alcohool drinking" and "adolescent" as descriptors. It included articles published between 2005 and 2014 that analyzed the association between alcohol consumption and physical violence using multivariate analysis. Of the total of 1667 articles located, 29 met the inclusion criteria. Alcohol consumption was investigated more as a predictor of involvement in physical violence when the teenager is the perpetrator or the victim of violence, with a significant association found in 19 studies. However, when victimization was investigated as a predictor (7 studies), most of these (6) revealed no significant association with the intake of alcoholic beverages. The consumption of alcohol has proven to be the predictor of physical violence for both the teen perpetrator and for the adolescent victim of violence. However, having been the victim of violence in childhood and adolescence may also lead adolescents to alcohol consumption.
\end{abstract}

Key words Violence, Alcoholic beverage consumption, Adolescent
Resumo O objetivo desta revisão integrativa foi investigar na literatura a associação entre consumo de bebidas alcoólicas e violência física, com ênfase em identificar o preditor entre eles. Foram realizadas buscas nas bases de dados Lilacs, $\mathrm{Me}$ dline e SciELO, adotando como descritores: "violence", "alcohool drinking" e "adolescent". Foram incluídos artigos publicados entre 2005 e 2014, e que analisassem a associação entre o consumo de álcool e a violência física, usando análise multivariada. Do total de 1667 artigos, 29 se enquadravam nos critérios de inclusão. O consumo de álcool foi a variável mais investigada como preditora do envolvimento em violência física, quando o adolescente é o perpetrador ou a vítima da violência, com associação significativa em 19 estudos. No entanto, quando a vitimização foi investigada como preditora (7 estudos), na maioria destes (6) houve associação significativa com a ingestão de bebidas alcoólicas. O consumo de bebida alcoólica se mostrou preditor da violência física, tanto para $o$ adolescente perpetrador quanto para aquele vítima da violência. Entretanto, ter sido vítima de violência na infância e na adolescência também pode levar o adolescente ao consumo do álcool.

Palavras chave Violência, Consumo de bebidas alcoólicas, Adolescente 


\section{Introdução}

O álcool é a droga mais consumida entre os adolescentes e representa um grave problema de saúde pública mundial ${ }^{1,2}$. Estudos demonstram que a prevalência do consumo de álcool entre essa população pode variar entre $15 \%$ e $51 \%$ nas Américas $^{2}$, e entre 2,6\% e 15,6\%, na Ásia ${ }^{3}$. No Brasil, apesar de proibida a venda de bebidas alcoólicas para menores de 18 anos, o consumo de álcool pelos jovens é uma prática comum e que se inicia cada vez mais cedo ${ }^{4}$.

O uso de álcool pode causar danos ao organismo quando ingerido por tempo prolongado. Além das consequências diretas da intoxicação, existem aquelas a longo prazo que podem interferir na vida do adolescente, como no aprendizado, na perspectiva de emprego, em comportamentos de risco no trânsito ${ }^{5}$, situações de violência, como envolvimento em brigas, violência sexual e doméstica, dentre outras ${ }^{1,6,7}$.

Estudar a relação entre violência e o consumo de bebidas alcoólicas envolve uma séries de complexidades pois as interligações são múltiplas e variadas. Todavia, estudos apontam que o consumo de álcool é, no mínimo, um importante fomentador de situações violentas, tanto sob o ponto de vista do agressor como da vítima ${ }^{8}$.

Em estudos epidemiológicos, a violência física aparece em posição de destaque no que se refere à saúde dos adolescentes, com maior prevalência no sexo masculino $0^{1,3,5,9,10}$, podendo estar relacionada ao ambiente doméstico ou escolar ${ }^{11}$. Existe maior vulnerabilidade do indivíduo a outros fatores de risco quando a violência doméstica é frequente e quando há vitimização durante a infância ${ }^{12}$.

A associação entre o consumo de bebidas alcoólicas e a violência física é documentada na literatura ${ }^{2,3,6}$, entretanto, afim de traçar estratégias de prevenção voltadas para esses fatores de risco. E para que essas estratégias sejam mais efetivas, é importante saber como essa relação acontece, identificando o preditor do problema. Então, seria a vitimização na infância, preditora do consumo de álcool ou o consumo de álcool favorece o envolvimento em episódios de violência entre os adolescentes? O objetivo deste estudo foi investigar na literatura se o consumo do álcool é preditor ou desfecho da violência física.

\section{Método}

Esta revisão integrativa da literatura procurou identificar artigos publicados nos últimos dez anos (2005 a 2014) na Biblioteca Virtual em Saúde (BVS) do Centro Latino-Americano e do Caribe de Informação em Ciências da Saúde, também conhecido pelo seu nome original, Bireme, e na US National Library of Medicine National Institutes of Health (PubMed). As bases de dados consultadas nessas bibliotecas foram: Lilacs, $\mathrm{Me}$ dline e SciELO.

Durante as etapas de seleção foram adotados como critérios de inclusão estudos com adolescentes entre 10 e 19 anos, que tratassem da associação entre a violência física e o consumo de álcool através de análise multivariada, que destacassem os pontos de corte e como as questões foram apresentadas nos questionários de coleta. $\mathrm{Na}$ análise dos dados nos estudos, entendeu-se como variável preditora, a variável independente ou explicativa.

Os critérios de exclusão foram os estudos de revisão de literatura e que não disponibilizassem o resumo nas bases de dados pesquisadas. A seleção dos artigos foi realizada em seis etapas: cruzamento dos descritores (1.667 artigos); aplicação dos filtros (968 artigos); leitura dos títulos (401 artigos); leitura dos resumos (197 arquivos); exclusão dos repetidos (52) e leitura integral dos artigos (29). A seleção dos artigos foi conduzida por dois revisores individualmente e os casos divergentes foram analizados em conjunto, baseando-se nos critérios de inclusão e exclusão até se chegar num consenso. A busca foi conduzida em inglês utilizando os descritores: "adolescence", "adolescent", "violence" e "alcohol drinking". Os filtros aplicados diziam respeito ao ano de publicação (2005 até 2014) e idioma da publicação (inglês, português e espanhol).

A extração dos dados dos artigos selecionados ocorreu a partir do método utilizado pelos autores. As variáveis de estudo relacionadas ao consumo de álcool e envolvimeto em situações de violência foram classificadas como dependentes ou independentes. A seguir, os valores correspondentes às razões de prevalência (RP), Odds Ratio (OR), beta $(\beta)$ e os seus respectivos intervalos de confiança foram extraídos dos resultados e analisados como significativos ou não.

\section{Resultados}

Dos 29 artigos, $72,4 \%(\mathrm{n}=21)$ foram estudos transversais e $27,6 \%(n=8)$ são estudos longitudinais. Nos estudos transversais o consumo de álcool foi o mais investigado como variável independente $(n=19)$. Quando a violência foi investigada como variável indepentende, os resultados 
também encontraram associação significativa. Já nos estudos longitudinais a violência foi mais investigada como preditora (variável independente).

Nos estudos transversais, abordou-se o envolvimento do adolescente em violência física sob duas perspectivas diferentes: o adolescente como vítima e como perpetrador da violência física.

Dos estudos transversais que abordaram o adolescente como vítima da violência física ( $\mathrm{n}=$ 11), quatro não encontraram associação significativa entre o consumo de álcool (variável independente) e a violência física ${ }^{4,6,13,14}$. Seis artigos apontaram o consumo de álcool como preditor da violência física (OR entre 1,31 e 10,25) 2,13,15-18. Em dois estudos, os resultados demonstraram que ser vítima de violência física na infância leva o indivíduo ao consumo de álcool quando adolescente (OR entre 1,23 e 3,36 ${ }^{12,13}$ ) (Tabela 1).

Dos 16 estudos que abordaram o adolescente como perpetrador da violência física, todos adotaram a violência como variável dependente. Destes, 11 artigos encontraram associação significativa para o consumo de bebidas alcoólicas como preditor (variável independente) para o envolvimento em violência física ${ }^{1,2,5,6,14,15,19-23}$. O OR variou de 1,5 a 6,89 (Tabela 2).

Nos estudos longitudinais $(\mathrm{n}=8)$, o tempo de acompanhamento dos sujeitos envolvidos, no geral, variou de 1 a 14 anos, exceto o estudo de Green et al. ${ }^{24}$, que acompanhou indivíduos por 36 anos. A vitimização como variável independente foi adotada por 05 autores ${ }^{9,11,25-27}$ (Tabela 3). Em seu estudo, White et al. ${ }^{28}$ optaram por analisar os dois sentidos dessa associação usando como variável independente tanto o consumo de álcool como a violência física e encontrou associação significativa para ambos. Dentre esses estudos, a maioria concluiu que ser vítima de violência física em algum determinado momento anterior da vida é preditor para o consumo de álcool entre os adolescentes ${ }^{9,11,25,27,28}$. Apenas dois estudos apontaram o consumo anterior de bebidas alcoólicas como preditor do envolvimento em situações de violência física ${ }^{7,28}$.

\section{Discussão}

A maioria $(65,5 \%)$ dos estudos incluídos nessa revisão apresentaram delineamento transversal e indicaram que o consumo de bebida alcoólica foi analisado como preditor para o envolvimento em situações de violência na adolescência. Para os estudos que abordaram o adolescente como vítima, os que consumiram álcool nos últimos 30 dias apresentaram, em média, duas vezes mais chan- ces de serem vítimas de violência do que adolescentes que não o consumiram ${ }^{2,15}$. Essa chance aumenta em até 13 vezes à medida que a quantidade de álcool ingerida também aumenta. Como também ocorre aumento da chance de vitimização nos casos de binge drinking (consumo de 5 ou mais doses de bebida alcoólica num determinado momento), de alcoolemia e de consumo diário de álcool ${ }^{15,16,18}$. Os estudos que adotaram o binge drinking como ponto de corte, encontraram associação positiva em maior frequência do que os que adotaram outros pontos de corte para o consumo do álcool ${ }^{4,5,21-23}$.

Outro aspecto que se pode destacar, foi a idade em que ocorreu o primeiro contato do adolescente com a bebida alcoólica. Beber em idade precoce $(<12$ anos) dobra o risco de ser uma vítima de violência em comparação com aqueles que nunca beberam ${ }^{13}$. Esse início precoce também aparece relacionado com posterior abuso de álcool e dependência na adolescência ${ }^{19}$.

Em contrapartida, dois estudos transversais que delinearam o consumo de álcool como variável dependente expuseram que a violência física pode ser preditora para o consumo de álcool. Indivíduos que sofreram maus-tratos na infância, em geral por familiares, apresentaram maiores chances de se tornarem consumidores de bebidas alcoólicas na adolescência ${ }^{12,19}$. Em seu estudo, Hamburger et al. ${ }^{19}$ sugerem, como justificativa, que a infância é um período do desenvolvimento em que os pais exercem grandes influencias sobre as crianças. Sendo assim, maus tratos nessa fase da vida pode trazer consequências no futuro, como o desenvolvimento de condutas de risco à saúde, a exemplo da ingestão de substâncias tóxicas como o álcool.

Quando o adolescente sai da posição de vítima e passa a ser analisado nos estudos como o perpetrador da violência, a associação significativa entre o álcool e o comportamento violento foi verificada em grande parte dos estu$\operatorname{dos}^{1-3,10,14,15,20,21,23,29}$. Isso pode ocorrer pelo fato dos adolescentes, sob efeito da bebida, perderem o senso crítico e se tornarem agressivos ${ }^{10}$.

No estudo de Swahn e Donovan ${ }^{7}$, os autores verificaram que no ato da violência física o indivíduo se encontrava sob efeito de bebida alcoólica, caracterizando a coocorrência destes comportamentos de risco. Este foi o único estudo que permitiu essa avaliação de forma direta, onde o álcool e a violência estavam presentes ao mesmo tempo no evento descrito ${ }^{7}$.

Outro aspecto avaliado foi a relação de gêneros, quando o adolescente é o perpetrador da violência. Os meninos apresentam maiores chan- 
Tabela 1. Descrição dos estudos transversais que abordam o adolescente como vítima de violência física, 2015.

\begin{tabular}{|c|c|c|c|c|}
\hline Autor/Ano & $\begin{array}{l}\text { Faixa } \\
\text { etária }\end{array}$ & Variável Independente & Variável dependente & $\mathrm{OR} / \mathrm{RP}(\mathrm{IC})$ \\
\hline \multirow[t]{2}{*}{$\begin{array}{l}\text { Shepherd et al. }{ }^{15} / \\
2006\end{array}$} & \multirow[t]{2}{*}{$\begin{array}{l}11-16 \\
\text { anos }\end{array}$} & $\begin{array}{l}\text { Consumo de álcool nos últimos } 30 \\
\text { dias }\end{array}$ & \multirow[t]{2}{*}{$\begin{array}{l}\text { Vítima de violência } \\
\text { física no último ano }\end{array}$} & $\begin{array}{l}\mathrm{OR}=2.94 \\
(2.19-3.95)\end{array}$ \\
\hline & & Ter ficado bêbado no último ano & & $\begin{array}{c}\mathrm{OR}=4.01 \\
(3.17-5.08\end{array}$ \\
\hline \multirow[t]{3}{*}{$\begin{array}{l}\text { Ramisetty-Mikle S } \\
\text { et al. }{ }^{13} / 2006\end{array}$} & \multirow[t]{3}{*}{$\begin{array}{c}13-19 \\
\text { anos }\end{array}$} & Binge drinking nos últimos 30 dias & \multirow{3}{*}{$\begin{array}{l}\text { Vítima de violência } \\
\text { física nos últimos } 12 \\
\text { meses }\end{array}$} & $\begin{array}{l}\mathrm{OR}=1.2 \\
(0.5-2.5)\end{array}$ \\
\hline & & $\begin{array}{l}\text { Primeiro contato com bebida } \\
\text { alcoólica na pré-adolescencia }\end{array}$ & & $\begin{array}{l}\mathrm{OR}=2.2 \\
(1.3-3.8)\end{array}$ \\
\hline & & $\begin{array}{l}\text { Consumo de álcool nos últimos } 30 \\
\text { dias }\end{array}$ & & $\begin{array}{l}\mathrm{OR}=1.0 \\
(0.4-2.6)\end{array}$ \\
\hline \multirow[t]{2}{*}{$\begin{array}{l}\text { Frederiksen et al. }{ }^{18} \\
/ 2007\end{array}$} & \multirow[t]{2}{*}{$\begin{array}{c}15-17 \\
\text { anos }\end{array}$} & Consumo diário de álcool & \multirow[t]{2}{*}{$\begin{array}{l}\text { Vítima de violência } \\
\text { física no último ano }\end{array}$} & $\begin{array}{l}\text { Masculino: } \\
4.1(1.8-9.1)\end{array}$ \\
\hline & & Consumo Semanal de álcool & & $\begin{array}{l}\text { Masculino: } \\
1.4(0.8-2.5)\end{array}$ \\
\hline \multirow[t]{3}{*}{$\begin{array}{l}\text { Hamburger et al. }{ }^{19} \\
\text { / } 2008\end{array}$} & \multirow[t]{3}{*}{-} & \multirow[t]{3}{*}{$\begin{array}{l}\text { Vítima de violência física na } \\
\text { infância, por familiares }\end{array}$} & $\begin{array}{l}\text { Consumo de álcool na } \\
\text { vida }\end{array}$ & $\begin{array}{c}\mathrm{OR}=1.90 \\
(1.56-2.30)\end{array}$ \\
\hline & & & $\begin{array}{c}\text { Primeiro contato com } \\
\text { bebida alcoólica na pré- } \\
\text { adolescência }\end{array}$ & $\begin{array}{l}\mathrm{OR}=2.10 \\
(1.69-2.63)\end{array}$ \\
\hline & & & $\begin{array}{l}\text { Binge drinking no } \\
\text { último ano }\end{array}$ & $\begin{array}{l}\mathrm{OR}=1.22 \\
(0.98-1.51)\end{array}$ \\
\hline Yen CF et al. ${ }^{12} / 2008$ & $\begin{array}{c}13-18 \\
\text { anos }\end{array}$ & $\begin{array}{l}\text { Vítima de violência física na } \\
\text { infância, por familiares }\end{array}$ & $\begin{array}{c}\text { Consumo de álcool nos } \\
\text { últimos } 30 \text { dias }\end{array}$ & $\begin{array}{c}\mathrm{OR}=3.360 \\
(2.389-4.725)\end{array}$ \\
\hline $\begin{array}{l}\text { Swahn MH et al. }{ }^{4} \\
2008\end{array}$ & $\begin{array}{c}12-16 \\
\text { anos }\end{array}$ & $\begin{array}{l}\text { Primeiro contato com bebida } \\
\text { alcoólica na pré-adolescência }\end{array}$ & $\begin{array}{l}\text { Vítima de violência } \\
\text { física no último ano }\end{array}$ & $\begin{array}{c}\mathrm{OR}=1.20 \\
(0.84-1.73)\end{array}$ \\
\hline \multirow[t]{2}{*}{ Yan FA et al. ${ }^{16} / 2010$} & \multirow[t]{2}{*}{$\begin{array}{c}13-18 \\
\text { anos }\end{array}$} & Consumo de álcool no último ano & \multirow[t]{2}{*}{$\begin{array}{l}\text { Vítima de violência } \\
\text { física no último ano }\end{array}$} & $\begin{array}{c}\mathrm{OR}=10.25 \\
(4.96-21.15)\end{array}$ \\
\hline & & Binge drinking no último ano & & $\begin{array}{c}\mathrm{OR}=13.54 \\
(6.21-29.51)\end{array}$ \\
\hline $\begin{array}{l}\text { Chaveepojnkamjorn } \\
\text { W, Pichainarong } \mathrm{N}^{6} \\
\text { / } 2011\end{array}$ & $\begin{array}{c}15-17 \\
\text { anos }\end{array}$ & $\begin{array}{l}\text { Consumo de álcool nos últimos } 30 \\
\text { dias }\end{array}$ & $\begin{array}{l}\text { Foi ameaçado ou } \\
\text { agredido com uma arma } \\
\text { nos últimos } 30 \text { dias }\end{array}$ & $\begin{array}{c}\mathrm{OR}=1.14 \\
(0.53-2.46)\end{array}$ \\
\hline $\begin{array}{l}\text { Whiteside LK et al. }{ }^{14} \\
\text { / } 2013\end{array}$ & $\begin{array}{c}14-18 \\
\text { anos }\end{array}$ & $\begin{array}{l}\text { Uso indevido do álcool no último } \\
\text { ano* }^{*}\end{array}$ & $\begin{array}{c}\text { Agressor e vítima de } \\
\text { violência no último ano }\end{array}$ & $\begin{array}{l}\mathrm{OR}=0.99 \\
(0.60-1.62)\end{array}$ \\
\hline $\begin{array}{l}\text { Pierobon } \mathrm{M} \text { et al. } \\
\text { / } 2014\end{array}$ & $\begin{array}{l}13-15 \\
\text { anos }\end{array}$ & $\begin{array}{l}\text { Consumo de álcool nos últimos } 30 \\
\text { dias }\end{array}$ & $\begin{array}{l}\text { Vítima de violência } \\
\text { física no último ano }\end{array}$ & $\begin{array}{c}\mathrm{OR}=2,32 \\
(1,63-3,31)\end{array}$ \\
\hline $\begin{array}{l}\text { Russell M et al. }{ }^{17} / \\
2014\end{array}$ & - & Binge drinking nos últimos 3 meses & $\begin{array}{c}\text { Vítima de violência } \\
\text { física nos últimos } 3 \\
\text { meses }\end{array}$ & $\begin{array}{l}\text { Feminino } \\
\text { OR }=1.31 \\
(1.10-1.57) \\
\end{array}$ \\
\hline
\end{tabular}

${ }^{*}$ Obtido através do POSIT (Problem Oriented Screening Instrument for Teenagers), utiliza uma escala até 17 pontos para avaliar situações de consumo indevido de álcool como faltar à escola, envolvimento em acidentes de carro após ingestão de álcool, dentre outros.

ces de se envolverem em situações de violência quando consomem bebidas alcoólicas do que as meninas ${ }^{1,21,23,29}$. Isso ocorre, provavelmente, pelas alterações comportamentais causadas por substâncias psicoativas, como o álcool, e pela maior frequência de consumo de álcool e de envolvimento em violência pelos meninos, em relação às meninas ${ }^{1}$.
O beber em binge, que representa o consumo de 4 ou mais doses de bebidas alcoólicas para meninas e 5 ou mais doses para os meninos, também aparece associado à perpetração da violência entre adolescentes, com Odds Ratio semelhantes ao do consumo atual de álcool. Entretanto, nenhum dos estudos, que adotou o beber em binge como variável independente, fez a distinção correspon- 
Tabela 2. Descrição dos estudos transversais que abordam o adolescente como perpetrador da violência física, 2015.

\begin{tabular}{|c|c|c|c|c|}
\hline Autor/Ano & $\begin{array}{l}\text { Faixa } \\
\text { etária }\end{array}$ & Variável Independente & Variável dependente & OR/RP (IC) \\
\hline \multirow[t]{2}{*}{ Swahn M, Donovan J / 2005} & \multirow[t]{2}{*}{$\begin{array}{c}12-21 \\
\text { anos }\end{array}$} & $\begin{array}{l}\text { Binge drinking nos últimos } 30 \\
\text { dias }\end{array}$ & \multirow{2}{*}{$\begin{array}{c}\text { Envolvimento em violência } \\
\text { física por estar bêbado no } \\
\text { último ano }\end{array}$} & $\begin{array}{c}\mathrm{OR}=2,63 \\
(1,72-4,02)\end{array}$ \\
\hline & & $\begin{array}{l}\text { Consumo de álcool nos últimos } \\
30 \text { dias }\end{array}$ & & $\begin{array}{c}\mathrm{OR}=2.18 \\
(1.17-4.03)\end{array}$ \\
\hline Shepherd JP et al. ${ }^{15} / 2006$ & $\begin{array}{l}11-16 \\
\text { anos }\end{array}$ & $\begin{array}{l}\text { Ter ficado bêbado no último ano } \\
\text { Consumo de álcool nos últimos } \\
30 \text { dias }\end{array}$ & $\begin{array}{l}\text { Perpetrador de violência } \\
\text { física no último ano no } \\
\text { último ano }\end{array}$ & $\begin{array}{c}\mathrm{OR}=2.10 \\
(1.84-2.41) \\
\mathrm{OR}=6.89 \\
(5.00-9.49)\end{array}$ \\
\hline \multirow[t]{4}{*}{ Stafström M M / 2007} & \multirow[t]{4}{*}{-} & $\begin{array}{l}\text { Binge drinking uma vez ao mês } \\
\text { ou com maior frequência }\end{array}$ & \multirow{4}{*}{$\begin{array}{l}\text { Envolvimento em brigas } \\
\text { relacionadas ao Consumo de } \\
\text { álcool (alguma vez na vida) }\end{array}$} & $\begin{array}{c}\mathrm{OR}=1.5 \\
(1.05-2.09)\end{array}$ \\
\hline & & $\begin{array}{l}\text { Ingestão de cerveja duas vezes ao } \\
\text { mês ou com maior frequência }\end{array}$ & & $\begin{array}{c}\mathrm{OR}=1.4 \\
(0.98-2.00)\end{array}$ \\
\hline & & $\begin{array}{l}\text { Ingestão de bebidas destiladas } \\
\text { duas vezes ao mês ou com maior } \\
\text { frequência }\end{array}$ & & $\begin{array}{c}\mathrm{OR}=1.7 \\
(1.16-2.35)\end{array}$ \\
\hline & & $\begin{array}{l}\text { Volume usual de cerveja excedeu } \\
1,5 \mathrm{l}\end{array}$ & & $\begin{array}{c}\mathrm{OR}=1.6 \\
(1.14-2.14)\end{array}$ \\
\hline Rudatsikira E et al. ${ }^{24} / 2008$ & $\begin{array}{c}15-17 \\
\text { anos }\end{array}$ & $\begin{array}{l}\text { Uso de substâncias } \\
\text { (álcool, maconha e tabaco) }\end{array}$ & $\begin{array}{l}\text { Perpetrador de violência } \\
\text { física no último ano } \\
\text { no último ano }\end{array}$ & $\begin{array}{c}\text { Feminino: } \\
1.82 \\
(1.47-2.26) \\
\text { Masculino: } \\
2.22(1.80-2.74)\end{array}$ \\
\hline Swahn MH et al. ${ }^{4} / 2008$ & $\begin{array}{l}12-16 \\
\text { anos }\end{array}$ & $\begin{array}{l}\text { Primeiro contato com bebidas } \\
\text { alcoólicas na pré-adolescencia }\end{array}$ & $\begin{array}{l}\text { Perpetrador de violência } \\
\text { física no último ano } \\
\text { no último ano }\end{array}$ & $\begin{array}{c}\mathrm{OR}=1.34 \\
(0,91-1.96)\end{array}$ \\
\hline Walton MA et al. ${ }^{22} / 2009$ & $\begin{array}{c}14-18 \\
\text { anos }\end{array}$ & Binge drinking no último ano & $\begin{array}{l}\text { Perpetrador de violência } \\
\text { física no último ano }\end{array}$ & $\begin{array}{c}\mathrm{OR}=1.72 \\
(1.06,2.81)\end{array}$ \\
\hline $\begin{array}{l}\text { Chaveepojnkamjorn W, } \\
\text { Pichainarong } \mathrm{N}^{6} / 2011\end{array}$ & $\begin{array}{c}15-17 \\
\text { anos }\end{array}$ & $\begin{array}{l}\text { Consumo de álcool nos últimos } \\
30 \text { dias }\end{array}$ & $\begin{array}{l}\text { Perpetrador de violência } \\
\text { física nos últimos } 30 \text { dias }\end{array}$ & $\begin{array}{c}\mathrm{OR}=3.06 \\
(1.99-4.70)\end{array}$ \\
\hline Andrade SSCA et al. ${ }^{1} / 2012$ & $\begin{array}{c}13-15 \\
\text { anos }\end{array}$ & $\begin{array}{l}\text { Consumo de álcool nos últimos } \\
30 \text { dias }\end{array}$ & $\begin{array}{l}\text { Perpetrador de violência } \\
\text { física nos últimos } 30 \text { dias }\end{array}$ & $\begin{array}{c}\text { Feminino } \\
1,30(0,62-2,61) \\
\text { Masculino } \\
\mathbf{2 , 2 1}(\mathbf{1 , 1 2 - 4 , 3 9 )}\end{array}$ \\
\hline Donath C et al. ${ }^{5} / 2012$ & $\begin{array}{c}12-17 \\
\text { anos }\end{array}$ & $\begin{array}{l}\text { Binge drinking nos últimos } 30 \\
\text { dias }\end{array}$ & $\begin{array}{l}\text { Envolvimento em violência } \\
\text { física na escola }\end{array}$ & $\begin{array}{c}\mathrm{OR}=0.987 \\
(0.972-1.003)\end{array}$ \\
\hline Wahl S et al. ${ }^{30} / 2013$ & $\begin{array}{l}\text { Média } \\
15,6 \\
\text { anos }\end{array}$ & $\begin{array}{l}\text { Consumo de álcool nos últimos } \\
30 \text { dias }\end{array}$ & $\begin{array}{l}\text { Perpetrador de violência } \\
\text { física no último ano }\end{array}$ & $\begin{array}{c}\mathrm{OR}=1,14 \\
(0,52-2,48)\end{array}$ \\
\hline Whiteside LK et al. ${ }^{14} / 2013$ & $\begin{array}{c}14-18 \\
\text { anos }\end{array}$ & $\begin{array}{l}\text { Uso indevido do álcool no último } \\
\text { ano }^{*}\end{array}$ & $\begin{array}{l}\text { Agressor e vitima de } \\
\text { violência física no último ano }\end{array}$ & $\begin{array}{c}\mathrm{OR}=1.94 \\
(1.26-3.00)\end{array}$ \\
\hline Stickley A et al. $.^{21} / 2013$ & $\begin{array}{c}13-17 \\
\text { anos }\end{array}$ & $\begin{array}{l}\text { Binge drinking nos últimos } 30 \\
\text { dias }\end{array}$ & $\begin{array}{l}\text { Perpetrador de violência } \\
\text { física no último ano }\end{array}$ & $\begin{array}{l}\text { Feminino: } \\
6.1(3.9-9.3) \\
\text { Masculino } \\
4.0(2.2-7.4)\end{array}$ \\
\hline Manickam MA et al. ${ }^{3} / 2014$ & $\begin{array}{c}12-17 \\
\text { anos }\end{array}$ & $\begin{array}{l}\text { Consumo de álcool nos últimos } \\
30 \text { dias }\end{array}$ & $\begin{array}{l}\text { Perpetrador de violência } \\
\text { física no último ano }\end{array}$ & $\begin{array}{c}\mathrm{OR}=1.36 \\
(1.15-1.61)\end{array}$ \\
\hline Mat Hussin SF et al. ${ }^{10} / 2014$ & $\begin{array}{c}12-17 \\
\text { anos }\end{array}$ & $\begin{array}{l}\text { Consumo de álcool nos últimos } \\
30 \text { dias }\end{array}$ & $\begin{array}{l}\text { Perpetrador de violência } \\
\text { física no último ano }\end{array}$ & $\begin{array}{c}\mathrm{OR}=1.42 \\
(1.24-1.63)\end{array}$ \\
\hline Pierobon M et al. $.^{14} / 2014$ & $\begin{array}{c}13-15 \\
\text { anos }\end{array}$ & $\begin{array}{l}\text { Consumo de álcool nos últimos } \\
30 \text { dias }\end{array}$ & $\begin{array}{l}\text { Perpetrador de violência } \\
\text { física no último ano }\end{array}$ & $\begin{array}{l}\mathrm{OR}=2,35 \\
(1,53-3,60)\end{array}$ \\
\hline $\begin{array}{l}\text { Salas-Wright CP et al. }{ }^{20} / \\
2014\end{array}$ & $\begin{array}{c}12-14 \\
\text { anos }\end{array}$ & $\begin{array}{l}\text { Consumo de álcool nos últimos } \\
30 \text { dias }\end{array}$ & $\begin{array}{l}\text { Perpetrador de violência } \\
\text { física no último ano }\end{array}$ & $\begin{array}{c}\mathrm{OR}=2.35 \\
(1.45-3.79)\end{array}$ \\
\hline
\end{tabular}


Tabela 3. Estudos Longitudinais que abordam a associação entre consumo de álcool e violência física entre adolescentes, 2015.

\begin{tabular}{|c|c|c|c|c|}
\hline Autor/Ano & $\begin{array}{c}\text { Tempo de } \\
\text { acompanhamento }\end{array}$ & Variável Independente & Variável dependente & OR, RP ou $\beta$ (IC) \\
\hline \multirow[t]{2}{*}{$\begin{array}{l}\text { Pinchevsky GM } \\
\text { et al. }{ }^{25} / 2013\end{array}$} & \multirow[t]{2}{*}{3 anos } & \multirow[t]{2}{*}{$\begin{array}{l}\text { Vítima de Violência no } \\
\text { último ano ( aos } 12 \text { anos } \\
\text { de idade) }\end{array}$} & $\begin{array}{l}\text { No de dias em que } \\
\text { consumiu álcool } \\
\text { (aos } 15 \text { anos) }\end{array}$ & $\begin{array}{l}\text { RP Masculino: } 0,13 \\
\quad(\mathrm{p}>0,05) \\
\text { Feminino: } 0,11 \\
(\mathrm{p}>0,05)\end{array}$ \\
\hline & & & $\begin{array}{l}\text { No de dias em que } \\
\text { bebeu em binge (aos } \\
15 \text { anos) }\end{array}$ & $\begin{array}{l}\text { RP Masculino: } 0,11 \\
(\mathrm{p}>0,05) \\
\text { Feminino: } \mathbf{0 , 5 8} \\
(\mathbf{p}<\mathbf{0 , 0 1})\end{array}$ \\
\hline \multirow[t]{2}{*}{$\begin{array}{l}\text { White HR et al. }{ }^{28} \\
\text { / } 2013\end{array}$} & \multirow[t]{2}{*}{14 anos } & $\begin{array}{l}\text { Aumento do consumo de } \\
\text { álcool (entre } 13 \text { e } 18 \text { anos de } \\
\text { idade) }\end{array}$ & $\begin{array}{l}\text { Aumento do } \\
\text { comportamento } \\
\text { violento (entre } 13 \text { e } 18 \\
\text { anos de idade) }\end{array}$ & $\begin{array}{c}\beta=0,51 \\
(p<0,05)\end{array}$ \\
\hline & & $\begin{array}{l}\text { Aumento do comportamento } \\
\text { violento (entre } 13 \text { e } 18 \text { anos } \\
\text { de idade) }\end{array}$ & $\begin{array}{l}\text { Aumento do consumo } \\
\text { de álcool (entre } 13 \text { e } 18 \\
\text { anos de idade) }\end{array}$ & $\begin{array}{c}\beta=0,04 \\
(p<0.01)\end{array}$ \\
\hline $\begin{array}{l}\text { Cisler JM et al. }{ }^{11} \\
\text { / } 2012\end{array}$ & 5 anos & $\begin{array}{l}\text { Soma das vezes em que } \\
\text { sofreu violência física, sexual } \\
\text { ou testemunhou cenas de } \\
\text { violência (coleta } 1 \text { ) }\end{array}$ & $\begin{array}{l}\text { Binge drinking no } \\
\text { último ano (coleta 2) }\end{array}$ & $\begin{array}{c}\beta=0,08 \\
(p<0,01)\end{array}$ \\
\hline \multirow[t]{2}{*}{$\begin{array}{l}\text { Green KM et } \\
\text { al. }^{24} / 2011\end{array}$} & \multirow[t]{2}{*}{36 anos } & \multirow[t]{2}{*}{$\begin{array}{l}\text { Consumo de álcool aos } 16 \\
\text { anos }\end{array}$} & $\begin{array}{l}\text { Perpetrador de } \\
\text { violência aos } 32 \text { anos }\end{array}$ & $\begin{array}{l}\mathrm{OR}=1.23 \\
{[0.79,1.90]}\end{array}$ \\
\hline & & & $\begin{array}{l}\text { Perpetrador de } \\
\text { violência aos } 42 \text { anos }\end{array}$ & $\begin{array}{l}\mathrm{OR}=1.10 \\
{[0.54,2.24]}\end{array}$ \\
\hline \multirow[t]{2}{*}{$\begin{array}{l}\text { Swahn MH, } \\
\text { Donovan } \mathrm{JE}^{7} / \\
2005\end{array}$} & \multirow[t]{2}{*}{1 ano } & $\begin{array}{l}\text { Consumo de álcool nos } \\
\text { últimos } 30 \text { dias (coleta 1) }\end{array}$ & \multirow{2}{*}{$\begin{array}{l}\text { Envolvimento em } \\
\text { briga relacionada ao } \\
\text { consumo de álcool no } \\
\text { último ano (coleta 2) }\end{array}$} & $\begin{array}{l}\mathrm{OR}=2.18 \\
(1.17-4.03)\end{array}$ \\
\hline & & Binge drinking (coleta 1 ) & & $\begin{array}{c}\mathrm{OR}=2.63 \\
(1.72-4.02)\end{array}$ \\
\hline \multirow[t]{2}{*}{$\begin{array}{l}\text { Mills R et al. }{ }^{26} / \\
2014\end{array}$} & \multirow[t]{2}{*}{14 anos } & \multirow[t]{2}{*}{$\begin{array}{l}\text { Vítima de violência na } \\
\text { infância (aos } 5 \text { anos de idade) }\end{array}$} & $\begin{array}{l}\text { Consumo de álcool } \\
\text { uma vez na vida (aos } \\
14 \text { anos de idade) } \\
\end{array}$ & $\begin{array}{l}\mathrm{OR}=1.11 \\
(0.45,2.71)\end{array}$ \\
\hline & & & $\begin{array}{l}\text { Binge drinking na vida } \\
\text { ou ou Consumo de } \\
\text { álcool nos últimos } 30 \\
\text { dias (aos } 14 \text { anos de } \\
\text { idade) }\end{array}$ & $\begin{array}{l}\mathrm{OR}=0.61 \\
(0.08,4.58)\end{array}$ \\
\hline \multirow[t]{2}{*}{$\begin{array}{l}\text { Shin SH et al. }{ }^{9} / \\
2013\end{array}$} & \multirow[t]{2}{*}{7 anos } & \multirow[t]{2}{*}{$\begin{array}{l}\text { Vítima de violência física na } \\
\text { vida ( coleta } 3 \text { ) }\end{array}$} & $\begin{array}{l}\text { Consumo de álcool no } \\
\text { último ano (coleta } 1)\end{array}$ & $\beta=-0,21(P<0,01)$ \\
\hline & & & $\begin{array}{l}\text { Binge drinking no } \\
\text { último ano (coleta } 1)\end{array}$ & $\beta=0,08(p<0,001)$ \\
\hline $\begin{array}{l}\text { Shin SH et al. }{ }^{27} \\
\text { / } 2009\end{array}$ & 7 anos & $\begin{array}{l}\text { Vítima de violência física na } \\
\text { vida (coleta } 3 \text { ) }\end{array}$ & $\begin{array}{l}\text { Binge drinking no } \\
\text { último ano (coletas } 1 \text {, } \\
2 \text { e } 3)\end{array}$ & $\begin{array}{c}\mathrm{OR}=1.34 \\
(1.02,1.76)\end{array}$ \\
\hline
\end{tabular}

dente ao sexo e adotou como beber em binge o consumo de 5 ou mais doses para ambos. Esse fato pode representar uma subestimativa para o beber em binge entre as meninas e justificar os achados de Stickley et al. ${ }^{21}$, em que as meninas apresentaram mais chance de se envolver em violência física após beber em binge do que os meninos.

Já nos estudos longitudinais, observou-se que a violência na infância foi um preditor para 
o consumo do álcool com maiores chances para o sexo feminino. Os responsáveis apontados como perpetradores da violência, nesses casos, são os pais, demonstrando a vulnerabilidade desses adolescentes no seio familiar ${ }^{9,11,25,27,28}$.

Um ponto que chama a atenção é que a maioria dos estudos longitudinais consideraram a violência como variável explicativa para o consumo do álcool com associação significante. Além disso, observou-se que os adolescentes que foram vítimas de violência tendem a ingerir um volume maior de álcool em um curto intervalo de tempo $^{7,9,25,27}$. Na contramão, o estudo de Mills et al. ${ }^{26}$ foi o único dos longitudinais que não encontrou associação entre beber em binge e se envolver em violência física, uma vez que aferiu o beber em binge de forma diferente aos demais, agrupando à variável o consumo de 3 ou mais doses com frequência mensal.

Os achados de White observaram que, com o passar do tempo, quando o indivíduo aumenta a ingestão de bebidas alcoólicas, também há o aumento no envolvimento em situações de violência. Este fato demonstra que mudanças no padrão de ingestão de bebidas alcoólicas ao longo da vida adulta podem ser reflexo dos comportamentos agressivos que o indivíduo possuía na adolescência $^{28}$.

Algumas observações devem ser feitas quanto aos pontos de corte adotados por alguns estudos. Uma delas diz respeito à padronização do tempo anterior ao evento estudado. A violência avaliada pelos entrevistados nos últimos 12 meses foi relacionada ao consumo de álcool nos últimos 30 dias. Adotar essa variação na referência pode trazer comprometimento à análise dos estudos, uma vez que as relações temporais são diferentes e expõem os indivíduos ao viés de memória, visto que os estudos que consideraram os eventos nos últimos 12 meses encontraram menos associação significativa do que os que consideraram entre 1 e 3 meses $2,3,10,14,15,18,20,21,23$. Alguns autores optaram por agrupar algumas variáveis estudadas. Isso pode influenciar nos resultados encontrados. Por exemplo, Rudatsikira et al. ${ }^{29}$ agruparam as variáveis consumo de álcool, tabaco e maconha numa única variável (uso de substâncias), o que impossibilita fazer conclusões sobre a influência do consumo de álcool isoladamente ${ }^{24}$. Já o estudo de Cisler et al. ${ }^{11}$ agrupou a violência física com a violência sexual e o testemunho de episódios violentos. Isto aponta que não só a violência física como outros tipos de violência podem estar associadas ao beber em binge ${ }^{11}$. Também no estudo de Mills et al. ${ }^{26}$, não houve associação para a violência física isoladamente, mas quando esta foi incorporada a outros tipos de violência numa única variável, houve associação significativa. Os maus tratos de qualquer tipo, seja ele sexual, físico ou emocional, juntos, podem ser preditores para o consumo de álcool e para o binge drinking ${ }^{26}$.

\section{Considerações finais}

O consumo de álcool foi considerado como variavel explicativa na maioria dos estudos transversais entre adolescentes, sendo esses vítimas ou agressores, ao contrário dos achados em estudos longitudinais, evidenciando que a relação entre consumo do álcool e violência fisica ainda não se encontra claramente definida e que os diferentes métodos e tipos de estudo podem esclarecer melhor essa relação.

É importante salientar que alguns estudos avaliaram a associação entre álcool e violência com diferenças nas relações temporais, ou que agruparam variáveis ou ainda associaram tipos de violência, por exemplo violência física e sexual. Estas metodologias, em face da multiplicidade das referências impossibilitam chegar a conclusão sobre o consumo de álcool isoladamente em relação à violência.

Visto que essa revisão integrativa não identificou nenhum estudo em que o adolescente agressor fosse preditor para o consumo de álcool, seria interessante a realização de estudos nessa perspectiva, para elucidar de forma mais muninciosa como a violência física está relacionada ao consumo de álcool.

\section{Colaboradores}

A autora AP Carvalho e a coautora TC Silva foram responsáveis pela busca dos artigos e análise dos títulos, resumos e textos na íntegra para avaliação dos critérios de inclusão e exclusão e autoria do texto inicial. As coautoras PAM Valença e CFBF Santos atuaram na concepção do artigo e na primeira revisão textual e metodológica e as coautoras V Colares e VA Menezes foram responsáveis pela redação e revisão final do texto. 


\section{Referências}

1. Andrade SSCDA, Yokota RTDC, Sá NNB, Silva MMA, Araújo WN, Mascarenhas MDM, Malta DC. Relação entre violência física, consumo de álcool e outras drogas e bullying entre adolescentes escolares brasileiros. Cad Saude Publica 2012; 28(9):1725-1736.

2. Pierobon M, Barak M, Hazrati S, Jacobsen KH. Alcohol consumption and violence among Argentine adolescents. J Pediatr 2013; 89(1):100-107.

3. Manickam MA, Abdul Mutalip MHB, Hamid HABA, Bt Kamaruddin R, Sabtu MYB. Prevalence, Comorbidities, and Cofactors Associated With Alcohol Consumption Among School-Going Adolescents in Malaysia. Asia-Pacific J Public Heal 2014; 26(Supl. 5):91S-99S.

4. Swahn MH, Bossarte RM, Sullivent EE. Age of alcohol use initiation, suicidal behavior, and peer and dating violence victimization and perpetration among high-risk, seventh-grade adolescents. Pediatrics 2008; 121(2):297-305.

5. Donath C, Graessel E, Baier D, Pfeiffer C, Bleich S, Hillemacher T. Predictors of binge drinking in adolescents: ultimate and distal factors - a representative study. BMC Public Health 2012; 12(1):263.

6. Chaveepojnkamjorn W, Pichainarong N. Current drinking and health-risk behaviors among male high school students in central Thailand. BMC Public Health 2011; 11(1):233

7. Swahn $\mathrm{MH}$, Donovan JE. Predictors of fighting attributed to alcohol use among adolescent drinkers. Addict Behav 2005; 30(7):1317-1334.

8. Duailibi S, Laranjeira R. Políticas públicas relacionadas às bebidas alcoólicas. Rev Saude Publica 2007; 41(5):839-848.

9. Shin SH, Miller DP, Teicher MH. Exposure to childhood neglect and physical abuse and developmental trajectories of heavy episodic drinking from early adolescence into young adulthood. Drug Alcohol Depend. 2013; 127(1-3):31-38.

10. Mat Hussin SF, Abd Aziz NS, Hasim H, Sahril N. Prevalence and Factors Associated With Physical Fighting Among Malaysian Adolescents. Asia-Pacific J Public Heal 2014; 26(Supl. 5):108S-115S.

11. Cisler JM, Begle AM, Amstadter AB, Resnick HS, Danielson CK, Saunders BE, Kilpatrick DG. Exposure to interpersonal violence and risk for PTSD, depression, delinquency, and binge drinking among adolescents: Data from the NSA-R. J Trauma Stress 2012; 25(1):33-40.

12. Yen CF, Yang MS, Chen CC, Yang MJ, Su YC, Wang MH, Lan CM. Effects of childhood physical abuse on depression, problem drinking and perceived poor health status in adolescents living in rural Taiwan. Psychiatry Clin Neurosci 2008; 62(5):575-583.

13. Ramisetty-Mikler S, Goebert D, Nishimura S, Caetano R. Dating violence victimization: Associated drinking and sexual risk behaviors of Asian, native Hawaiian, and caucasian high school students in Hawaii. J Sch Health 2006; 76(8):423-429.

14. Whiteside LK, Ranney ML, Chermack ST, Zimmerman MA, Cunningham RM, Walton MA. The overlap of youth violence among aggressive adolescents with past-year alcohol use-A latent class analysis: aggression and victimization in peer and dating violence in an inner city emergency department sample. J Stud Alcohol Drugs 2013; 74(1):125-135.

15. Shepherd JP, Sutherland I, Newcombe RG. Relations between alcohol, violence and victimization in adolescence. J Adolesc 2006; 29(4):539-553.
16. Yan FA, Howard DE, Beck KH, Shattuck T, Hallmark-Kerr M. Psychosocial correlates of physical dating violence victimization among Latino early adolescents. J Interpers Violence 2010; 25(5):808-331.

17. Russell M, Cupp PK, Jewkes RK, Gevers A, Mathews C, LeFleur-Bellerose C, Small J. Intimate partner violence among adolescents in Cape Town, South Africa. Prev Sci 2014; 15(3):283-295.

18. Frederiksen ML, Helweg-Larsen K, Larsen HB. Self-reported violence amongst adolescents in Denmark: Is alcohol a serious risk factor? Acta Paediatr Int J Paediatr 2008; 97(5):636-640.

19. Hamburger ME, Leeb RT, Swahn MH. Childhood maltreatment and early alcohol use among high-risk adolescents. J Stud Alcohol Drugs 2008; 69(2):291-295.

20. Salas-Wright CP, Hernandez L, R. Maynard B, Y. Saltzman L, Vaughn MG. Alcohol Use Among Hispanic Early Adolescents in the United States: An Examination of Behavioral Risk and Protective Profiles. Subst Use Misuse 2014; 49(7):864-877.

21. Stickley A, Koyanagi A, Koposov R, Razvodovsky Y, Ruchkin V. Adolescent binge drinking and risky health behaviours: Findings from northern Russia. Drug Alcohol Depend 2013; 133(3):838-844.

22. Walton MA, Cunningham RM, Goldstein AL, Chermack ST, Zimmerman MA, Bingham CR, Shope JT, Stanley R, Brow FC. Rates and Correlates of Violent Behaviors Among Adolescents Treated in an Urban Emergency Department. J Adolesc Heal. 2009; 45(1):77-83.

23. Stafström M. Kick back and destroy the ride: alcohol-related violence and associations with drinking patterns and delinquency in adolescence. Subst Abuse Treat Prev Policy 2007; 2:18.

24. Green KM, Doherty EE, Zebrak KA, Ensminger ME Association between adolescent drinking and adult violence: evidence from a longitudinal study of urban african americans. J Stud Alcohol Drugs 2011; 72(5):701-710.

25. Pinchevsky GM, Wright EM, Fagan AA. Gender differences in the effects of exposure to violence on adolescent substance use. Violence Vict 2013; 28(1):122-144.

26. Mills R, Alati R, Strathearn L, Najman JM. Alcohol and tobacco use among maltreated and non-maltreated adolescents in a birth cohort. Addiction 2014; 109(4):672-680

27. Shin SH, Edwards EM, Heeren T. Child abuse and neglect: Relations to adolescent binge drinking in the national longitudinal study of Adolescent Health (AddHealth) Study. Addict Behav 2009; 34(3):277-280.

28. White HR, Fite P, Pardini D, Mun EY, Loeber R. Moderators of the dynamic link between alcohol use and aggressive behavior among adolescent males. J Abnorm Child Psychol 2013; 41(2):211-222.

29. Rudatsikira E, Mataya RH, Siziya S, Muula AS. Association between bullying victimization and physical fighting among Filipino adolescents: Results from the global school-based health survey. Indian J Pediatr 2008; 75(12):1243-1247.

30. Wahl S, Sonntag T, Roehrig J, Kriston L, Berner MM. Characteristics of predrinking and associated risks: A survey in a sample of German high school students. Int J Public Health 2013; 58(2):197-205

Artigo apresentado em 30/11/2015

Aprovado em 10/08/2016

Versão final apresentado em 12/08/2016 